\title{
Two-PHoton Excitation in Functional BIOLOGICAL IMAGING
}

\author{
Winfried Denk \\ Bell Laboratories, Lucent Technologies, Biological Computation Research Department, Murray Hill, \\ New Jersey 07974 \\ (Paper JBO-074R received Nov. 10, 1995; revised manuscript received March 25, 1996; accepted for publication May 13, \\ 1996)
}

Keywords nonlinear optics; $\mathrm{Ca}^{++}$imaging; brain slices; Ti:sapphire, fluorescence; uncaging; light scattering; laser scanning; confocal microscopy.

\section{INTRODUCTION}

Optical methods have always played an important role in the study of physiological phenomena. Light microscopes have been used for more than one hundred years ${ }^{1}$ to gain insight into the structure of biological systems in general and the nervous system in particular. Anatomical studies had given us a first understanding of shapes and types of nerve cells. ${ }^{2}$ Another example is the ubiquitous and rarely mentioned use of the light microscope to guide electrophysiological recording electrodes toward their targets. Only in the last two decades, however, has light microscopy become widely used to gather quantitative dynamic information about physiological processes. The list of examples includes: the study of diffusion in membranes with fluorescence methods; ${ }^{3-6}$ the detection of transmembrane potential changes using intrinsic signals ${ }^{7}$ or extrinsic fluorophores (for reviews see Refs. 8, 9, and 10); the measurements of nanometer displacements; ${ }^{11-14}$ and the quantification of changes in intracellular ion concentration using fluorescent indicator dyes (for a review see Ref. 15). More recently, light has also been used to trigger physiological processes by applying biological effector molecules with high spatial and temporal resolution via photochemical release, commonly called "uncaging."16-19 In this article, I review the contribution that the combination of two-photon absorption with laser scanning ${ }^{20}$ has made to functional biological imaging in recent years.

\section{TWO-PHOTON EXCITATION: HISTORY AND BACKGROUND}

Two-photon excitation is not a new idea. However, after being theoretically predicted ${ }^{21}$ in 1931 as a consequence of the then newly discovered and mathematically formalized quantum mechanics, it took 30 years before an experimental confirmation was obtained. ${ }^{22}$ The reason for this long delay is the miniscule size of typical two-photon absorption cross sections, which render two-photon excitation virtually nonexistent under ambient light condi- tions. Even at the light intensity present inside the arc of a high-pressure mercury-vapor lamp (the brightest conventional light source), only about one two-photon absorption event occurs per fluorophore every one hundred seconds. This is the absolute upper limit for conventional light sources since, as a result of the brightness theorem, light cannot be concentrated beyond the source intensity (see Ref. 23, Sec. 4.8.3). The laser, on the other hand, when operating in a single lateral mode, allows the concentration of all its power into a single diffraction-limited spot. This has permitted the study of excited electronic states by two-photon absorption spectroscopy (for a review see Ref. 24).

An early proposal for two-photon excitation microscopy involved a resonant cavity, ${ }^{25}$ which is, however, impractical for most applications and still suffers from the presence of a very high average power density at the sample. The development of the cw-mode-locked laser ${ }^{26-28}$ finally provided, at moderate average powers, molecular excitation rates that are large enough $\left(10^{8} / \mathrm{s}\right.$ for a single fluorophore, which is the saturation limit) to be useful for laser scanning microscopy. ${ }^{20}$ While two-photon excited laser scanning images can be obtained with cW lasers, ${ }^{29,30}$ the speed of image acquisition at the same average power is about 100,000 times slower, which makes the study of dynamic physiological phenomena virtually impossible. Two-photon absorption by cw beams can, however, become important as a damage mechanism during optical trapping experiments. ${ }^{31-33}$

\section{USING FLUORESCENCE FOR PHYSIOLOGICAL MEASUREMENTS}

\subsection{ONE EXAMPLE: MEASURING $\mathrm{CA}^{2+}$ CONCENTRATION}

One of the most important applications of light to gather quantitative physiological data has been the use of fluorescent indicators to measure the dynamics of the free calcium concentration inside cells. ${ }^{15,34-37}$ The main reason for the importance of calcium ion influx is that it serves almost univer- 
sally to couple membrane depolarization, which is detected by voltage-sensitive $\mathrm{Ca}^{++}$-permeable ion channels (for an excellent introduction see Ref. 38), with biochemical processes, which are often dependent on calcium binding proteins. ${ }^{39,40}$ Crucial to functional fluorescence microscopy are highly sensitive instruments such as cooled CCD devices, ${ }^{35,36}$ or laser scanning confocal ${ }^{41,42}$ and two-photon microscopes. $^{20,43-49}$

\subsection{IMAGING IN SCATTERING TISSUE}

With widefield illumination and focal plane detection (intensified TV and CCD detectors), high resolution imaging inside intact tissue is severely hampered by light scattering, which can be particularly strong in nervous tissue, and by fluorescence background resulting from exogenous or endogenous fluorophores. In confocal microscopy, unwanted out-of-focus and scattered light is rejected by the detector pinhole. Two major obstacles remain: photodamage, also referred to as phototoxicity, and photobleaching (P\&P). In fact, P\&P can even be exacerbated, compared to, say, cooled CCD detection, as a result of the smaller detector quantum efficiency for photomultipliers and, more fundamentally, due to the loss of fluorescence photons that originate at the focus but are scattered in the tissue and then miss the detector pinhole (Fig. 1-see color plate on p. 301). In addition, while information is only collected for the focal slice, excitation and hence P\&P occur throughout the sample.

\subsection{CONFOCAL VS TWO-PHOTON}

The P\&P problems encountered with confocal microscopy can be solved while retaining excellent optical sectioning capabilities by using two-photon excitation of fluorescence. ${ }^{20,43,44,46}$ Because optical sectioning occurs during excitation alone, ${ }^{48}$ twophoton excited laser scanning microscopy (TPLSM) does not require confocal detection. A further consequence of the well-localized excitation is that P\&P do not occur outside the focal slice, ${ }^{48}$ as was convincingly demonstrated first by Piston and Webb, ${ }^{4-46}$ who compared directly the one-photon and the two-photon case, and, more recently, by Potter et al. ${ }^{49}$

Another important advantage ${ }^{43}$ of using twophoton excitation is that its longer excitation wavelengths are scattered less ${ }^{50,51}$ and hence penetrate deeper into the tissue (remember that we are using about twice the wavelength compared to onephoton excitation of the same fluorophore). This property of infrared light is also exploited in the visualization of living brain slices ${ }^{52,53}$ and to some extent in diffusive-wave imaging. ${ }^{54}$ An experimental confirmation of the improved depth penetration was obtained recently by Potter et al. ${ }^{49}$ and by Centonze et al., ${ }^{55}$ who compared directly on the same samples two-photon (non-confocal) and onephoton confocal microscopy.
Finally, as discussed later in detail, scattered fluorescence photons can contribute to the signal without any loss of spatial resolution. This is because in TPLSM, unlike in a confocal microscope, the detection process does not need to contribute to resolution or background rejection. ${ }^{43}$ Scattered excitation light, on the other hand, is rendered harmless because it is diluted. As a practical matter, the comparative simplicity of the optical path when wholearea detection is used ${ }^{46}$ helps to increase further the efficiency with which fluorescent photons are captured.

\subsection{APPLICATIONS OF DEEP TISSUE IMAGING}

Imaging deep inside tissue is particularly important when intact and complete cells are to be studied within their original environment, for example, to address questions of neural circuitry. When imaging in living brain tissue slices, ${ }^{56}$ one traditionally had to make the choice between limited resolution ${ }^{57}$ or the need to select cells near the slice surface where the integrity of the local circuitry and the health of individual cells can be questionable. . $^{5,59}$ Functional confocal imaging has generally been plagued with photodamage problems. ${ }^{60}$ It has recently been demonstrated that TPLSM does overcome these problems and permits, for example, protracted imaging of synaptic spines, ${ }^{47,61}$ which are micron-sized protrusions from neuronal dendrites and serve as fundamental units of dendritic integration. Another example where depth penetration and damage minimization are crucial is imaging of developing embryos. ${ }^{62,63}$

\subsection{TWO-PHOTON EXCITATION IN SCATTERING TISSUE}

High-resolution functional imaging in scattering tissue may well be where the strengths of twophoton excited fluorescence imaging are most effective. ${ }^{43}$ As touched upon previously, imaging in scattering tissue can be accomplished by the use of either one-photon excitation combined with confocal detection or of two-photon excitation without the need for confocal detection. To compare both methods, several issues have to be considered ${ }^{43}$ (Fig. 1). Scattering (1) considerably reduces the amount of excitation light that reaches the focus and (2) generates a diffuse background of excitation light. While one can compensate for the light loss at the focus by increasing the incident power, this has very different consequences for one- and twophoton excitation. In the one-photon case, increased P\&P occur in the excitation path and throughout the diffuse background. Neither effect is significant for two-photon excitation, since the sharp (quadratic) drop of the absorption rate with the light intensity precludes significant excitation away from the focus. In addition, the diffuse background, which comprises light arriving along different path- 
lengths, is temporally less coherent, thus reducing two-photon absorption further, at least for excitation with subpicosecond pulses. Another issue is the reduction of the effective numerical aperture, since light with a high transverse momentum, i.e., propagating at a large angle to the optical axis, traverses more tissue and thus suffers greater extinction. This effect is more severe for the shorter, more strongly scattered wavelengths that are used for one-photon excitation, resulting in a loss not only of excitation efficiency but also of resolution.

On the emission side, the fraction of fluorescence light that leaves the ample as "ballistic" photons (photons that are not scattered) is greatly reduced, as the depth of tissue that has to be traversed increases. In a confocal microscope, only ballistic photons can pass the detector pinhole and contribute to the signal, "diffusive" photons are lost. But every photon generated by a fluorophore, detected or not, uses up the valuable capital of a limited number of excitation events that organic fluorophores can undergo before suffering photodestruction. ${ }^{64}$ In other words, to achieve a given signal-to-noise ratio under scattering conditions with a confocal microscope, a larger fraction of the available fluorophores will be destroyed and a larger amount collateral photodynamic damage to the biological tissue will be incurred.

In a two-photon excitation microscope, both ballistic photons and diffusive photons (which can be the vast majority) contribute to the signal as long as nondescanned whole-field detection or descanned detection with a large enough detector ${ }^{46}$ is used. "Large enough" here means to be roughly of the size of the scattering halo, which, in turn, has a diameter on the order of the imaging depth. As mentioned before, optical sectioning, equivalent to that of an ideal confocal microscope (with infinitesimal pinhole size $\mathrm{e}^{5}$, results from the quadratic dependence ${ }^{48,66,67}$ (but note Ref. 68) of the excitation probability on the local light intensity.

Thus in scattering samples two-photon excitation reduces $\mathrm{P} \& \mathrm{P}$ significant even in the focal plane, while fully retaining its out-of-focus advantage already familiar from optically clear samples. ${ }^{20,46}$ For example, the out-of-focus reduction of P\&P amounts to a factor of roughly 1000 at a distance $\Delta z=10 \mu \mathrm{m}$ from the focal plane when imaging with a 1.3 NA objective lens. The reason ${ }^{48}$ is that in a laser scanning microscope the time averaged light intensity is roughly independent of $\Delta z$. In the case of one-photon excitation, P\&P are therefore not confined to the focal slice. In fact, they are almost independent of $\Delta z$ (see e.g., Fig. 3 in Ref. 46). The average two-photon excitation rate and any excitation-linked damage, on the other hand, fall off quickly as the average of the squared instantaneous intensity falls off $\left(\propto \Delta z^{-2}\right.$ for $\left.\Delta z \gg \lambda / \mathrm{NA}^{2}\right)$.

\subsection{TWO-PHOTON EXCITATION WITH CONFOCAL DETECTION}

It is a quite natural idea to use a confocal detection pinhole in conjunction with two-photon excitation. ${ }^{48}$ Such a combination has been implemented and characterized experimentally by Stelzer et al. ${ }^{69}$ The benefits are significantly improved resolution and some additional background rejection. For physiology experiments, in particular when imaging in scattering tissue, these benefits have to be weighted carefully against the drawbacks: (1) using a pinhole that is small enough to provide significant resolution improvements ${ }^{65}$ also entails, even for nonscattering samples, a significant loss of fluorescence light; ${ }^{46}(2)$ the large wavelength difference between excitation and emission is likely to exacerbate problems resulting from lateral chromatic aberration, which, unless stage scanning is used, where optical axis always passes through the imaged pixel, leads to a misalignment of excitation and detection volumes; and (3) in scattering samples only ballistic (unscattered) fluorescence photons can contribute to the signal (see above).

\subsection{WHERE IS TWO-PHOTON EXCITATION IMAGING APPROPRIATE AND NECESSARY?}

The reduction and confinement of photodamage and photobleaching available with two-photon excitation is particularly relevant under the following circumstances: (1) whenever images at multiple focal planes have to be acquired such as for threedimensional reconstruction of morphology; (2) when damage load on the whole tissue has to be reduced, for example when imaging in brain slices $^{47,61}$ in developing tissue where damage to the genetic material has to be minimized to preserve the ability of the cells to divide properly, ${ }^{62,63,70}$ or when imaging intrinsic functional signals with low fluorescence yield, e.g., redox imaging using NAD (P) $H_{1}^{71,72}$ (3) when, even while imaging only a single focal plane, an out-of-focus pool of diffusible fluorophore exists that can replace those lost by bleaching, for example, when imaging cells filled with intracellular indicators. ${ }^{47,61,73,74}$

\section{4 Рнотоstimulation}

Another major use of light in physiology lies in stimulating a biological system rather than merely observing it. In the simplest case, photoreceptors are illuminated and a physiological response is observed. To stimulate with light when there is no intrinsic responsivity requires the introduction of a "sensitizer." One example are molecules that change their biological efficacy via a photochemical reaction either reversibly (by photo isomerization) or irreversibly (by photolytic cleavage). ${ }^{16,75-81}$ Such "caged" molecules have become readily available 
during the last two decades and are now widely used to deliver effector molecules either when very high time resolution (below a millisecond) is required $^{82}$ or when diffusional access is slow, for which the intracellular space is an extreme example. ${ }^{83,84}$

Some spatial localization of release can be achieved optically, ${ }^{18,85,86}$ but release with high spatial resolution is difficult for several reasons: (1) most caged compounds require UV light for their activation, which makes high resolution release expensive, even in optically clear specimens, since special laser light sources and microscope optics are required; ${ }^{87}$ (2) scattering, which is particularly pronounced at shorter wavelengths, makes it next to impossible to release with high resolution deep inside, for example, a brain slice; (3) release always occurs throughout the whole focus cone, ${ }^{88}$ which makes true localization along all three spatial dimensions almost impossible. An additional aspect ${ }^{89}$ of photostimulation is that, unlike in confocal fluorescence microscopy, the detection process, using, e.g., transmembrane current ${ }^{18,85,89}$ often cannot be exploited to provide additional resolution, optical sectioning, and background rejection.

\subsection{TWO-PHOTON ABSORPTION MEDIATED PHOTOCHEMISTRY}

For the same reasons that have been discussed above for fluorescence imaging, two-photon absorption provides true 3-D localization (i.e., optical sectioning) using the excitation process alone. Furthermore, at twice the one-photon wavelength, two-photon excitation avoids the need to use special objective lenses, even for photolysis energies in the UV. Two-photon excitation using red light has been demonstrated to uncage ATP ${ }^{20}$ and caged fluorophores. ${ }^{90,91}$ The highly localized release of caged carbamoylcholine has been used to map neurotransmitter-receptor distributions on cultured cells. ${ }^{92}$ A remaining problem is that for currently available caged compounds, two-photon action cross sections for the release of agonist are typically more than 1000 times smaller (about 0.001-0.010 $\left.\mathrm{GM}^{93,94}\right)$; than those for the excitation of fluorescence in common fluorophores such as fluorescein or rhodamine, which have cross sections between $5-100$ GM. ${ }^{67}$ It is worth mentioning here that twophoton cross sections cannot be calculated by simply rescaling the corresponding one-photon spectrum (discussed in more detail in Ref. 46 and in references cited therein). Small two-photon cross sections then leave only a narrow range where the excitation power is sufficient for release and still below the tissue damage threshold. ${ }^{92,94}$ Intrinsic absorption, in addition, is much more severe, in part due to two-photon absorption by $\mathrm{NAD}(\mathrm{P}) \mathrm{H}^{72}$ for the wavelengths that are required for two-photon uncaging $^{92}(<700 \mathrm{~nm})$ than for the wavelengths $(830-860 \mathrm{~nm})$ used, for example, for functional $\mathrm{Ca}^{++}$ imaging in brain slices. ${ }^{47,61}$

Finally, combining two-photon photobleaching or two-photon photorelease of fluorescence with twophoton fluorescence imaging allows the measurement of diffusional transport with high resolution inside scattering tissue. In this way the diffusional and electrical coupling between synaptic spines and their parent shafts has recently been measured. ${ }^{91}$

\section{OPEN QUESTIONS}

Many of the physical parameters relevant for twophoton excitation are not yet fully understood. For example, reliable and precise measurements of absolute two-photon cross sections for some common fluorophores are only now appearing in the literature. ${ }^{66,67}$ It is clear that two-photon excitation minimizes chromophore excitation, which, independent of the excitation pathway (one- or multiphoton absorption), always carries a finite probability of causing a damaging reaction. However, it is unclear whether and to what degree there are limits on the application of two-photon excitation that are imposed by damage mechanisms that depend on nonlinear processes driven by the high peak intensities present in tightly focused ultrashort pulses. The practical experience ${ }^{20,47,49,61,63,74,95,96}$ has been that quite extensive imaging at high resolution and high signal-to-noise ratios is possible without loss of physiological function. This has been demonstrated particularly clearly by the extended observation of synaptic transmission in single dendritic spines $^{47,61}$ and by the protracted high resolution imaging of sea urchin embryos going through many cell division cycles. ${ }^{63}$

\subsection{POTENTIAL PROBLEMS}

When imaging thin ( 1 to $2 \mu \mathrm{m})$ nonscattering samples, two-photon excitation is not expected to possess fundamental advantages over one-photon confocal or widefield microscopy, since no fluorescence photons are lost due to scattering and, for a given excitation rate per fluorophore, bleaching in the focal plane should be comparable for both methods. Surprises, in favor or disfavor of TPLSM, might result from differences in photophysics and photochemistry between one- and two-photon molecular excitation. For example, due to the difference in excitation wavelength, one- and two-photon absorption by the excited state ${ }^{97}$ is bound to be different. Unintentional two-photon absorption by aromatic amino acids ${ }^{98,99}$ might even be responsible for some of the damage during high-intensity onephoton imaging but might be avoided using TPLSM. Similarly, during two-photon imaging, unintentional three-photon absorption ${ }^{5,100-102}$ may become a source of tissue damage. However, since the two-photon absorption rate $R_{n}$ is proportional to $\tau P_{\text {peak }}$ one can, by adjusting peak power $\left(P_{\text {peak }}\right)$ and 
pulselength $(\tau)$ appropriately reduce the rate of unwanted three or more photon processes without a the same time reducing the excitation efficiency for the desired two-photon processes. When the highest possible resolution is desired, the longer excitation wavelength of two-photon excitation is a disadvantage, but confocal detection is addition to two-photon excitation ${ }^{45}$ can provide a remedy that is, not, however, without its own drawbacks (see Sec. 3.6). One practical problem with two-photon imaging is that optical sectioning is not controllable simply by opening a detector pinhole, making small isolated fluorescent objects more difficult to find.

\subsection{PROMISING DIRECTIONS}

Endogenous fluorophores ${ }^{99}$ often pose a particular challenge to fluorescence imaging since they are not designed for minimal P\&P as many synthetic fluorophores are. While still in its infancy, two-photon imaging of the intrinsic metabolic indicator NAD $(\mathrm{P}) \mathrm{H}^{72}$ could become a useful tool for the study of dynamic physiological processes with high spatial resolution. Imaging or locally inactivating proteins with multiphoton excitation ${ }^{103}$ is largely unexplored. Two-photon micro-photopharmacology has been demonstrated, ${ }^{92}$ but for a breakthrough into widespread applications, compounds with larger two-photon cross sections are needed. Fluorescent lifetime imaging is easy to implement, ${ }^{104}$ since the excitation light is already pulsed. Fluorescence decay time contrast may become useful for the calibration of calcium indicators that change their excited state lifetime but not their excitation and emission spectra on binding of $\mathrm{Ca}^{++} .105$

Single fluorophore detection is clearly possible in purified biochemical samples, ${ }^{106-108}$, but in a cellular environment it is severely hampered by fluorescence and by Raman scattering from intrinsic chromophores. Two-photon excitation ${ }^{109}$ may allow better discrimination against excitation light and reduce background for two reasons: (1) Raman scattering is negligible because detection occurs far in the antistokes regime and (2) two-photon absorption by weak intrinsic fluorophores, present at high concentration, might be strongly reduced compared to the one-photon case. The reason is that twophoton absorption cross sections depend on the fourth power of the typical transition matrix element between molecular electronic states, but the one-photon cross section depends only on the square (see Ref. 110, p. 64).

Similar considerations apply to fluorescence correlation spectroscopy, ${ }^{6,111,112}$ which may well be resuscitated by two-photon excitation. ${ }^{113}$

Using three-photon absorption allows the excitation of fluorophores, such as amino and nucleic acids and some $\mathrm{Ca}^{++}$indicators, with excitation energies in the UV while still using the wavelengths obtainable from Ti:sapphire lasers ${ }^{100-102}$ and Nd:YLF. ${ }^{113 a}$

In more complicated optical arrangements such as $4 \mathrm{Pi}^{114,115}$ and theta microscopy, ${ }^{69}$ two-photon excitation $^{30}$ can either serve to avoid having to align a confocal detection path or it can be used to gain additional resolution and background rejection. By apodizing the illumination beam appropriately, the excitation volume can be reshaped: for example, using an annular aperture creates a volume elongated along the optical axis but with slightly narrower lateral extent. ${ }^{116}$ Since the full objective aperture can be used for detection in this case, the aperture entails no loss of fluorescent photons, quite different from the case of annular aperture confocal microscopy. ${ }^{117}$ By using nonlinear depletion or stimulated emission processes, far-field superresolution might be achievable. ${ }^{118,119}$

\section{OUTLOOK}

The major obstacles hindering the spread of twophoton excited microscopy have been the expense and complexity of ultrashort-pulse lasers used for excitation. The commercial availability of the Ti: sapphire laser ${ }^{120}$ has eliminated the requirement to keep a "laser jock" at hand, but not the significant capital expense and utility requirements.

The emerging alternative are all-solid-state, diode-pumped lasers, ${ }^{121-127}$ which can be used to acquire two-photon laser scanning images. ${ }^{55,128}$ Not only will diode-pumped systems ultimately be much cheaper, but they also consume about 1000 times less electrical power, are easy to cool, and require less than a tenth of the optical table space. Commercial diode-pumped systems are about to become available and for some (if not most) applications, such systems will undoubtedly dominate eventually.

It has to be kept in mind, however, that in research applications flexibility is essential and the combination of abundant power, optimal pulsewidth, ${ }^{46}$ and broad tunability may make the Ti:sapphire laser the source of choice ${ }^{129}$ for quite some time to come. The recent arrival of a diodepumped frequency double Nd:YAG laser capable of adequately pumping the Ti:sapphire lasers has reduced the utility requirements but not yet the cost.

Where two-photon excitation will ultimately be applied, within and outside of physiology, will depend on its price and the degree of advantage it offers compared to other techniques. In the near future I see increasing use of two-photon excitation in those areas where it promises overwhelming advantages, i.e., where its depth penetration and lowphotodamage properties are crucial. $43,46,47,49,61,62,91$

\section{Acknowledgments}

I thank Karel Svoboda for many useful comments on the manuscript. 


\section{confocal 2-photon}

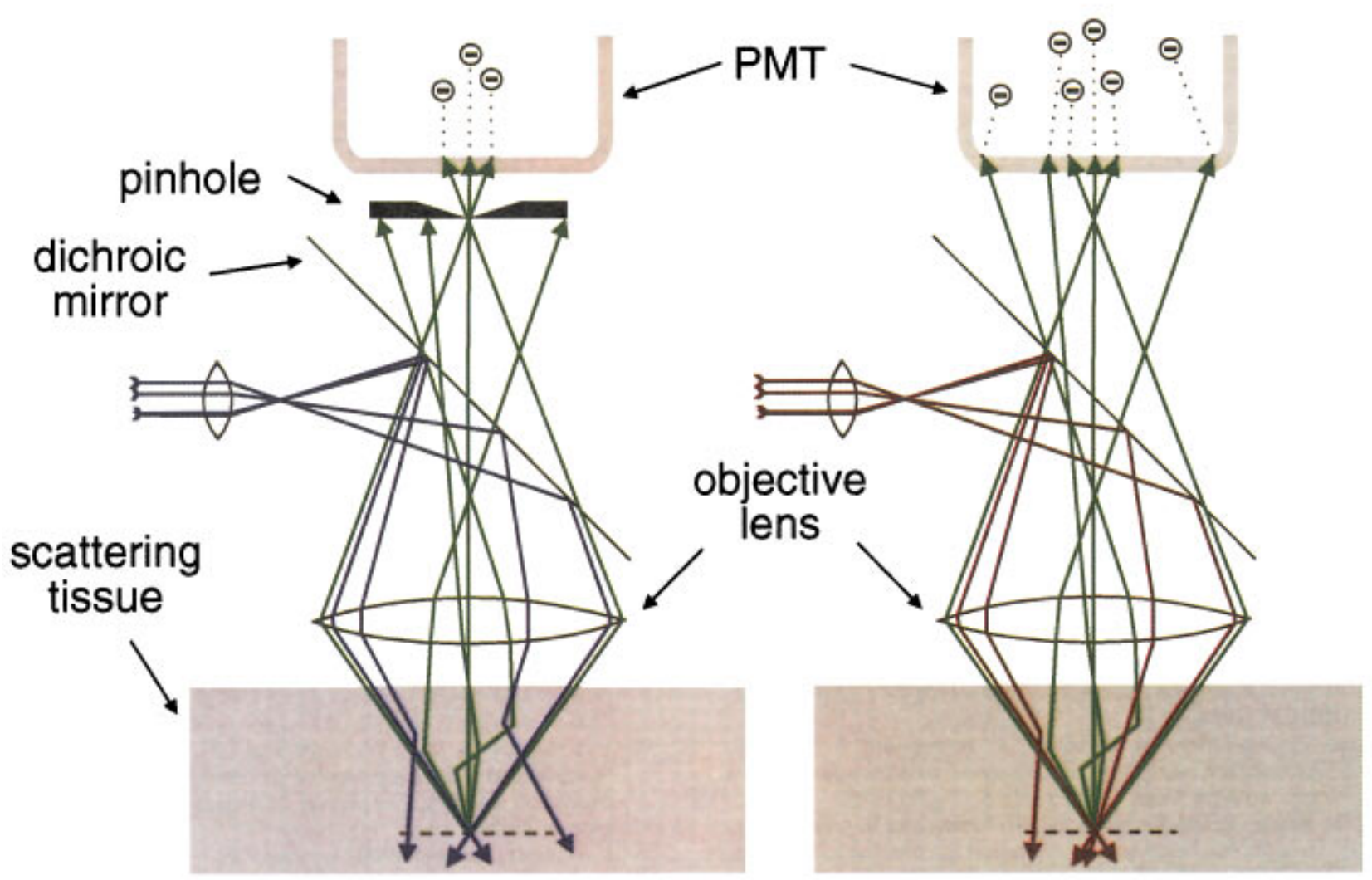

Fig. 1 Confocal one-photon excitation imaging compared with two-photon imaging in scattering tissue. Due to the longer wavelength, less excitation light is lost to scattering when using two-photon excitation. Ballistic and diffusing fluorescence photons can be used in the two-photon case, but only ballistic photons can be used in the confocal case.

\section{COLOR PLATE}

\section{REFERENCES}

1. S. Ramón y Cajal, "Significación fisiológica de las expansiones protoplásmicas y nerviosas de la sustancia gris," Revista de ciencias médicas de Barcelona 22, 23 (1891).

2. S. Ramón y Cajal, La Textura del Sistema Nerviosa del Hombre y los Vertebrados, Moya, Madrid (1904).

3. R. Peters, J. Peters, K. Tews, and W. Bahr, "A microfluorimetric study of translational diffusion in erythrocyte membranes," Biochim. Biophys. Acta. 367, 282-294 (1974).

4. D. Axelrod, D. E. Koppel, J. Schlessinger, E. Elson, and W. W. Webb, "Mobility Measurements by Analysis of Fluorescence Photobleaching Recovery Kinetics," Biophys. J. 16, 1055-1069 (1976).

5. E. A. Nigg and R. J. Cherry, "Anchorage of a band 3 population at the erythrocyte cytoplasmic membrane surface: protein rotational diffusion measurements," Proc. Natl. Acad. Sci. (USA) 77, 4702-4706 (1980).

6. E. Elson, "Fluorescence correlation spectroscopy and photobleaching recovery," Ann. Rev. Phys. Chem. 36, 379-406 (1985).

7. L. Cohen, R. Keynes, and B. Hille, "Light scattering and birefringence changes during nerve activity," Nature 218, 438-441 (1968).

8. L. Cohen, B. Salzberg, and A. Grinvald, "Optical methods for monitoring neuron activity," Ann. Rev. Neurosci. 1, 171$182(1978)$

9. A. Grinvald, R. D. Frostig, E. Lieke, and R. Hildesheim,
"Optical imaging of neuronal activity," Physiol. Rev. 68, 1285-1365 (1988).

10. L. Cohen, "Optical approaches to neuronal function," in $A n$ nual Review of Physiology, J. F. Hoffman and P. De Weer, Eds., pp. 487-582, Annual Review Inc., Palo Alto, CA (1989).

11. B. C. Hill, E. D. Schubert, and M. A. Nokes, "Laser interferometer measurement of changes in crayfish axon diameter concurrent with action potential," Science 196, 426-428 (1977).

12. J. Howard and A. Hudspeth, "Compliance of the hair bundle associated with giant of mechanoelectrical tranduction channels in the bullfrog's saccular hair cell," Neuron 1, 189-199 (1988).

13. W. Denk and W. W. Webb, "Optical measurements of picometer displacements of transparent microscopic objects," Appl. Opt. 29, 2382-2391 (1990).

14. K. Svoboda, C. F. Schmidt, B. J. Schnapp, and S. M. Block, "Direct observation of kinesin stepping by optical trapping interferometry," Nature 365, 721-727 (1993).

15. R. Y. Tsien, "Fluorescent probes of cell signaling," Ann. Rev. Neurosci. 12, 227-253 (1989).

16. H. A. Lester and H. W. Chang, "Response of acetylcholine receptors to rapid photochemically produced increases in agonist concentration," Nature 266, 373-374 (1977).

17. A. M. Gurney and H. A. Lester, "Light-flash physiology with synthetic photosensitive compounds," 67, 583-617 (1987).

18. E. M. Callaway and L. C. Katz, "Photostimulation using 
caged glutamate reveals functional circuitry in living brain slices," Proc. Natl. Acad. Sci. (USA) 90, 7661-7665 (1993).

19. J. W. Walker, J. A. McCray, and G. P. Hess, "Photolabile protecting groups for an acetycholine receptor ligand. Synthesis and photochemistry of a new class of o-nitrobenzyl derivatives and their effects on receptor function," Biochem. 25, 1799-1805 (1986)

20. W. Denk, J. H. Strickler, and W. W. Webb, "Two-photon laser scanning fluorescence microscopy," Science 248, 73-76 (1990).

21. M. Goeppert-Mayer, "Ueber elementarakte mit zwei quantenspruengen," Ann. Phys. 9, 273 (1931).

22. W. Kaiser and C. B. G. Garrett, "Two-photon excitation in $\mathrm{CaF}_{2}: \mathrm{Eu}^{2+}, "$ Phys. Rev. Lett. 7, 229-231 (1961).

23. M. Born and E. Wolf, Principles of Optics, Pergamon, New York (1980).

24. D. M. Friedrich and W. M. McClain, "Two-photon molecular spectroscopy," Ann. Rev. Phys. Chem. 31, 559-577 (1980).

25. C. J. R. Sheppard and R. Kompfner, "Resonant scanning optical microscope," Appl. Opt. 17, 2879-2882 (1978).

26. J. A. Giordmaine, P. M. Rentzepis, S. L. Shapiro, and K. W. Wecht, "Two-photon excitation of fluorescence by picosecond light pulses," Appl. Phys. Lett. 11, 216-218 (1967).

27. R. L. Fork, B. I. Greene, and C. V. Shank, "Generation of optical pulses shorter than $0.1 \mathrm{psec}$ by colliding pulse mode locking," Appl. Phys. Lett. 38, 671-672 (1981).

28. T. R. Gosnell and A. J. Taylor, eds., Selected Papers on Ultrafast Laser Technology, SPIE, Milestone Series, MS44, SPIE Press, Bellingham, WA (1991).

29. P. Hanninen, E. Soini, and S. Hell, "Continuous wave excitation two-photon fluorescence microscopy," J. Microsc. 176, 222-225 (1994)

30. P. E. Hanninen, M. Schrader, E. Soine, and S. W. Hell, "Two-photon excitation fluorescence microscopy using a semiconductor laser," Bioimaging 3, 70-75 (1995).

31. K. König, Y. Liu, G. Sonek, M. W. Berns, and B. J. Tromberg, "Two-photon fluorescence excitation in continuous wave infrared optical tweezers," Opt. Lett. 20, 2246-2248 (1995).

32. K. König, H. Liang, M. Berns, and B. Tromberg, "Cell damage by near-IR microbeams," Nature 377, 20-21 (1995).

33. K. König, Y. Liu, G. Sonek, and M. Berns, "Autofluorescence spectroscopy of optically trapped cells," Photochem. Photobiol. 62, 830-835 (1995).

34. G. Grynkiewicz, M. Poenie, and R. Y. Tsien, "A new generation of $\mathrm{Ca}^{2+}$ indicators with greatly improved fluorescence properties," J. Biol. Chem. 260, 3440-3450 (1985).

35. D. Williams, K. Fogarty, R. Tsien, and F. Fay, "Calcium gradients in single smooth muscle cells revealed by the digital imaging microscope using Fura-2," Nature 318, 558-561 (1985).

36. J. A. Connor, "Digital imaging of free calcium changes and of spatial gradients in growing processes in single, mammalian central nervous system cells," Proc. Natl. Acad. Sci. (USA) 83, 6179-6183 (1986).

37. A. Minta, J. P. Y. Kao, and R. Y. Tsien, “Fluorescent indicators for cytosolic calcium based on rhodamine and fluorescein chromophores," J. Biol. Chem. 264, 8171-8178 (1989).

38. B. Hille, Ionic Channels of Excitable Membranes, 2nd ed., Sinauer, Sunderland, MA (1992)

39. A. Crivici and M. Ikura, "Molecular and structural basis of target recognition by calmodulin," Ann. Rev. Biophys. Biomol. Struct. 24, 85-116 (1995).

40. H. Vogel, "The Merck Frosst Award Lecture 1994. Calmodulin: a versatile calcium mediator protein," Biochem. Cell. Biol. 72, 357-376 (1994).

41. M. Minski, "Microscopy apparatus," US patent 3013467 (1961).

42. T. Wilson and C. Sheppard, Theory and Practice of Scanning Optical Microscopy, Academic Press, New York (1984).

43. W. Denk, K. R. Delaney, A. Gelperin, D. Kleinfeld, B. W. Strowbridge, D. W. Tank, and R. Yuste, "Anatomical and functional imaging of neurones using 2-photon laser scanning microscopy," J. Neurosci. Meth. 54, 151-162 (1994).

44. R. M. Williams, D. W. Piston, and W. W. Webb, "Twophoton molecular excitation provides intrinsic 3-dimensional resolution for laser based microscopy and microphotochemistry," FASEB J. 8, 804-813 (1994).

45. E. H. K. Stelzer, S. Hell, and S. Lindek, "Nonlinear absorption extends confocal fluorescence microscopy into the ultra-violet regime and confines the illumination volume," Opt. Comm. 104, 223-228 (1994).

46. W. Denk, D. W. Piston, and W. W. Webb, "Two-photon molecular excitation in laser scanning microscopy," in The Handbook of Confocal Microscopy, J. Pawley, Ed., pp. 445-458, Plenum, New York (1995).

47. R. Yuste and W. Denk, "Dendritic spines as basic functional units of neuronal integration," Nature 375, 682-684 (1995).

48. W. Denk, J. H. Strickler, and W. W. Webb, "Two-photon laser scanning fluorescence microscopy," Science 248, 73-76 (1990).

49. S. Potter, C. M. Wang, P. Garrity, and S. Fraser, "Intravital imaging of green fluorescent protein using two-photon laser scanning microscopy," Gene 1995 (in press).

50. L. O. Svaasand and R. Ellingsen, "Optical properties of human brain," Photochem. Photobiol. 38, 293-299 (1983).

51. L. O. Svaasand, B. J. Tromberg, R. C. Haskell, T.-T. Tsay, and M. W. Berns, "Tissue characterization and imaging using photon density waves," Opt. Eng. 32, 258-266 (1993).

52. H. Dodt, G. Hager, and W. Zieglgansberger, "Direct observation of neurotoxicity in brain slices with infrared videomicroscopy," J. Neurosci. Meth. 50, 165-171 (1993).

53. H.-U. Dodt and W. Ziegelgänsberger, "Infrared videomicroscopy: a new look at neuronal structure and function," TINS 17, 453-458 (1994).

54. B. Chance, K. Hang, L. He et al., "Highly sensitive object location in tissue models with linear in-phase and antiphase multielement optical arrays ij one and $\mathrm{t} 2$ dimensions," Proc. Natl. Acad. Sci. (USA) 90, 3423-3427 (1993).

55. V. E. Centonze, D. L. Wokosin, and J. G. White, "Improved deep optical sectioning capabilities rendered by two-photon excitation imaging," Molecular Biology of the Cell 6, 657 (abstract) (1995).

56. J. Neurosci. Methods 59(1), 1-195 (1995).

57. D. W. Tank, M. Sugimori, J. A. Connor, and R. R. Llinás, "Spatially resolved calcium dynamics of mammalian Purkinje cells in cerebellar slice," Science 242, 773-777 (1988).

58. W. Müller and J. A. Connor, "Dendritic spines as individual neuronal compartments for synaptic $\mathrm{Ca} 2+$ responses," $\mathrm{Na}$ ture 354, 73-76 (1991).

59. R. Malinow, N. Otmakhov, K. I. Blum, and J. Lisman, “Visualizing hippocampal synatic function by optical detection of $\mathrm{Ca}^{2+}$ entry through the $\mathrm{N}$-methyl-D-aspatate channel," Proc. Natl. Acad. Sci. (USA) 91, 8170-8174 (1994).

60. S. Alford, B. G. Frenguelli, J. G. Schofied, and G. L. Collingridge, "Characterization of $\mathrm{Ca}^{2+}$ signals induced in hippocampal CA1 neurones by the synaptic activation of NMDA receptors," J. Physiol. (London) 469, 693-716 (1993).

61. W. Denk, M. Sugimori, and R. Llinas, "Two types of calcium response limited to single spines in cerebellar Purkinje cells," Proc. Natl. Acad. Sci. (USA) 92, 8279-8282 (1995).

62. R. G. Summers, J. B. Morrill, A. Lieth, M. Marko, D. W. Piston, and A. T. Stonbreaker, "A stereometric analysis of karyokinesis, cytokinesis and cell arrangements during and following fourth cleavage period in the sea urchin Lytechinus variegatus," Develop. Growth Differ. 35, 41-57 (1993).

63. R. G. Summers, D. W. Piston, K. M. Harris, and J. B. Morrill, "The orientation of the first cleavage in the sea urchin embryo, Lytechinus variegatus, does not specify the axes of bilateral symmetry," Dev. Biol. 175, 177-183 (1996).

64. S. Soper, H. Nutter, R. Keller, L. Davis, and E. Shera, "The photophysical constants of several fluorescent dyes pertaining to ultrasensitive fluorescence spectroscopy," Photochem. Photobiol. 57, 972-977 (1995).

65. M. Gu and C. J. R. Sheppard, "Effects of finite-sized detector on the OTF of confocal fluorescent microscopy," Optik 89, 65-69 (1991).

66. C. Xu, J. Guild, W. Webb, and W. Denk, "Determination of absolute two-photon excitation cross-sections by in situ second-order autocorrelation," Opt. Lett. 20, 2372-2374 (1995).

67. C. Xu and W. Webb, "Measurement of two-photon excitation cross sections of molecular fluorophores with data from 
690 to $1050 \mathrm{~nm}, "$ J. Opt. Soc. Am. B 13, 481-491 (1996).

68. A. Fischer, C. Cremer, and E. Stelzer, "Fluorescence of coumarins and xanthenes after two-photon absorption with a pulsed titanium sapphire laser," Appl. Opt. 34, 1989-2003 (1995).

69. E. H. K. Stelzer, S. Hell, and S. Lindek, "Nonlinear absorption extends confocal microscopy into the ultra-violet regime and confines the excitation volume," Opt. Comm. 104, 223-228.

70. D. W. Piston, R. G. Summers, and W. W. Webb, “Observation of nuclear division in living sea urchin embryos by twophoton fluorescence microscopy," Biophys. J. 63, A110 (1993).

71. B. Bennet, T. Jetton, G. Ying, M. Magnuson, and D. Piston "Quantitative subcellular imaging of glucose metabolism within intact pancreatic islets,"' J. Biol. Chem. 271, 3647-3651 (1995).

72. D. W. Piston, B. R. Masters, and W. W. Webb, "Threedimensionally resolved $\mathrm{NAD}(\mathrm{P}) \mathrm{H}$ cellular metabolic redox imaging of the in situ cornea with two-photon excitation laser scanning microscopy," J. Microscopy 178, 20-27 (1995).

73. D. W. Piston, M. S. Kirby, H. Cheng, W. J. Lederer, and W. W. Webb, "Two-photon-excitation fluorescence imaging of three-dimensional calcium-ion activity," Appl. Opt. 33, 662669 (1994).

74. W. Denk, J. R. Holt, G. M. G. Shepherd, and D. P. Corey, "Calcium imaging of single stereocilia in hair cells: localization of transduction channels at both ends of tip links," Neuron 15, 1311-1321 (1995).

75. H. A. Lester, M. E. Krouse, M. M. Nass, N. H. Wassermann, and B. F. Erlanger, "Light-activated drug confirms a mechanism of ion channel blockade," Nature 280, 509-510 (1979).

76. H. A. Lester and J. M. Nerbonne, "Physiological and pharmacological manipulations with light flashes," Ann. Rev. Biophys. Bioeng. 11, 151-175 (1982).

77. R. Y. Tsien and R. S. Zucker, "Control of cytoplasmic calcium with photolabile tetracarboxylate 2-nitrobenzhydrol chelators," Biophys. J. 50, 843-853 (1986).

78. J. A. McCray, L. Herbette, and T. Kihara, and D. R. Trentham, "A new approach to time-resolved studies of ATP-requiring biological systems: Laser flash photolysis of caged ATP," Proc. Natl. Acad. Sci. (USA) 77, 7237-7241 (1980).

79. J. E. T. Corrie and D. R. Trentham, "Caged nuceotids and neurotransmitters," in Bioorganic Photochemistry, H. Morrison, Ed., Wiley and Sons, New York (1993).

80. J. H. Kaplan, B. Forbush III, and J. F. Hoffman, "Rapid photolytic release and adenosine 5 '-triphosphate from a protected analogue: Utilization by the Na:K: pump of human red blood cell ghosts," Biochem. 10, 1929-1935 (1978).

81. G. Hess, L. Niu, and R. Wieboldt, "Determination of the chemical mechanism of neurotransmitter receptor mediated reactions by rapid chemical kinetic methods," Ann. NY Acad. Sci. 757, 4260-4263 (1995).

82. T. Milburn, N. Matsubara, A. P. Billington, J. B. Udgaonkar, J. W. Walker, B. K. Carpenter, W. W. Webb, J. Marque, W. Denk, J. A. McCray, and G. P. Hess, "Synthesis, photochemistry, and biological activity of a caged photolabile acetylcholine receptor ligand," Biochem. 28, 49-55 (1989).

83. K. Khodakhah and D. Ogden "Functional heterogeneity of calcium release by inositol triphosphate in single purkinje neurons, cultured cerebellar astrocytes, and peripheral tissues," Proc. Natl. Acad. Sci. (USA) 90, 4976-4980 (1994).

84. R. C. Malenka, J. A. Kauer, R. S. Zucker, and R. A. Nicoll, "Postsynaptic calcium is sufficient for potentiation of hippocampal synaptic transmission," Science 242, 81-84 (1988).

85. M. Dalva and L. Katz, "Rearrangements of synaptic connections in visual cortex revealed by laser photostimulation," Science 265, 255-258 (1994).

86. L. Katz and M. Dalva, "Scanning laser photostimulation: A new approach for analyzing brain circuits," J. Neurosci. Meth. 54, 205-218 (1994).

87. C. Bliton, J. Lechleiter, and D. E. Clapham, "Optical modifications enabling simultaneous confocal imaging with dyes excited by ultraviolet- and visible-wavelength light," J. Microsc. 169, 15-26 (1993).
88. S. S.-H. Wang and G. Augustine, "Confocal imaging and local photolysis of caged compounds: Dual probes of synaptic function," Neuron 15, 755-760 (1995).

89. W. Denk, "Two-photon laser scanning photochemical microscopy used to map the distribution of ligand gated ion channels," Soc. Neurosci. Abstr. 19, 91 (1993).

90. I. Silberzan, R. M. Williams, and W. W. Webb, "Fluorescence photoactivation by two-photon excitation: Kinetics of uncaging and three-dimensional point diffusion measurements," Biophys. J. 63, A109 (1993).

91. K. Svoboda, D. W. Tank, and W. Denk, "Direct measurement of coupling between dendritic spines and shaft," Science 272, 716-719 (1996).

92. W. Denk, "Two-photon scanning photochemical microscopy: mapping ligand-gated ion channel distributions," Proc. Natl. Acad. Sci. (USA) 91, 6629-6633 (1994).

93. J. Steyer and W. Denk, unpublished data.

94. K. Svoboda and W. Denk, unpublished results.

95. J. A. Ridsdale and W. W. Webb, "The viability of cultured cells under two-photon laser scanning microscopy," Biophys. J. 63, A109 (1993).

96. S. Potter, J. Pine, and S. Fraser, "Neural transplant staining with DiI and vital imaging by 2-photon laser-scanning microscopy," Scann. Micr. Intl. (suppl.) 1995 (in press).

97. Y. Yuan and D. Axelrod, "Subnanosecond polarized fluorescence photobleaching: Rotational diffusion of acetylcholine receptor on developing muscle cells," Biophys. J. 69, 690-700 (1995).

98. A. A. Rehms and P. R. Callis, "Two-photon fluorescence excitation spectra of aromatic amino acids," Chem. Phys. Lett. 208, 276-282 (1993).

99. Y.-W. Xu, J.-R. Zhang, Y.-M. Deng, L.-K. Hue, S.-P. Jiang, and S.-H. Lian, "Fluorescence of proteins induced by twophoton absorption," J. Photochem. Photobiol. B 1, 233-227 (1987).

100. I. Gryczynski, H. Szmacinski, and J. Lakowicz, "On the possibility of calcium imaging using INDO-1 with 3-photon excitation," Photochem. Photobiol. 62, 804-80 (1995).

101. I. Gryczynski, H. Malak, and J. Lakowicz, "3-Photon induced fluorescence of 2,5-diphenyloxazole with a femtosecond Ti sapphire laser," Chem. Phys. Lett. 245, 30-35 (1995).

102. W. Webb, personal communication (1995).

103. J. Liao, J. Roider, and D. Jay, "Chromophore assisted laser inactivation of proteins is mediated by the photogeneration of free radicals," Proc. Natl. Acad. Sci. (USA) 91, 2659-2663 (1994).

104. D. W. Piston, D. R. Sandison, and W. W. Webb, "Timeresolved fluorescence imaging and background rejection by two-photon excitation in laser scanning microscopy," Proc. SPIE 1640, 379-389 (1992).

105. J. R. Lakowicz, M. L. Szmanski, and M. L. Johnson, "Calcium imaging using fluorescence lifetimes and longwavelength probes," J. Fluorescence 2, 47 (1992).

106. R. Rigler, U. Mets, J. Widengren, and P. Kask, "Fluorescence correlation spectroscopy with high count rate and low background: Analysis of translational diffusion," European Biophys. J. 22, 169-175 (1993).

107. S. Soper, Q. Mattingly, and P. Vegunta, "Photon burst detection of single near infrared fluorescent molecules," Anal. Chem. 65, 740-747 (1993).

108. S. Nie, D. Chiu, and R. Zare, "Probing individual molecules with confocal fluorescence microscopy," Science 266, 1018-1021 (1994).

109. J. Mertz, C. Xu, and W. W. Webb, "Single molecule detection by two-photon excited fluorescence," Opt. Lett. 20, 2532-2535 (1996).

110. A. Yariv, "Thermodynamic fluctuations in a reacting system-measurement by fluorescence correlation spectroscopy," in Quantum Electronics, 3rd ed., p. 676, Wiley and Sons, New York (1989).

111. D. Magde, E. Elson, and W. W. Webb, Phys. Rev. Lett. 29, 705-708 (1972).

112. G. Feher and M. Weissman, "Fluctuation spectroscopy: Determination of chemical reaction kinetics from the fre- 
quency spectrum of fluctuations," Proc. Natl. Acad. Sci. (USA) 70, 870-875 (1973).

113. K. M. Berland, P. T. So, and E. Gratton, "Two-photon fluorescence correlation spectroscopy: method and application to the intracellular environment," Biophys. J. 68, 649-701 (1995).

113a. D. L. Wokosin, V. E. Centonze, S. Crittenden, and J. G. White, "3-Photon excitation of blue emitting fluorophores by laser scanning microscopy," Molecular Biology of the Cell 6, 656 (1995).

114. S. Hell and E. H. K. Stelzer, "Fundamental improvement of resolution with a $4 \mathrm{Pi}$-confocal fluorescence microscope using two-photon excitation," Optics Comm. 93, 277-282 (1992).

115. S. Hell, S. Lindek, and E. H. K. Stelzer, "4Pi-confocal microscopy," in 1993 International Conference on Confocal Microscopy and 3-D Image Processing, Sidney (1993).

116. S. Hell, P. Hanninen, A. Kuusisto, M. Schrader, and E. Soini, "Annular aperture two-photon excitation microscopy," Opt. Comm. 117, 20-24 (1995).

117. M. Gu and C. J. R. Sheppard, "Effects of annular pupils on confocal fluorescent imaging," J. Mod. Opt. 39, 1883-1896 (1992).

118. S. Hell and J. Wichmann, "Breaking the diffraction resolution limit by stimulated emission: stimulated emission depletion fluorescence microscopy," Opt. Lett. 19, 780-782 (1994).

119. S. Hell and M. Kroug, "Ground state depletion fluorescence microscopy: A concept for breaking the diffraction resolution limit," Appl. Phys. B 60, 495-497 (1995).

120. D. E. Spence, P. N. Kean, and W. Sibbett, "60-fsec pulse generation from a self-mode-locked Ti:sapphire laser," Opt. Lett. 16, 42-44 (1991).

121. F. Falcoz, F. Balembois, P. Georges, and A. Brun, "Self starting self mode locked femtosecond diode pumped $\mathrm{Cr}$ Lisaf laser," Opt. Lett. 20, 1874-1876 (1995).

122. S. Tsuda, W. H. Knox, E. de Souza, W. Jan, and J. Cunningham, "Low loss intracavity AlAs/AlGaAs saturable Bragg reflector for femtosecond mode locking in solid state lasers," Opt. Lett. 20, 1406-1408 (1995).

123. G. Lenz, K. Tamura, H. Haus, et al., "All solid state femtosecond source at $1.55 \mu \mathrm{m}, "$ Opt. Lett. 20, 1289-1291 (1995).

124. K. Lamb, D. Spence, J. Hong, et al., "All solid state self mode locked Ti sapphire laser," Opt. Lett. 19, 1864-1866 (1994).

125. M. Ober, M. Hofer, U. Keller, and T. H. Chiu, "Self-starting diode pumped femtosecond Nd fiber laser," Opt. Lett. 18, 1532-1534 (1993).

126. P. Mellish, P. French, J. Taylor, et al., "All solid state femtosecond diode pumped Cr:LiSAF laser," Electr. Lett. 30, 223-224 (1994).

127. U. Keller, "Ultrafast all solid state laser technology," Appl. Phys. B-Lasers and Optics 58, 347-363 (1994).

128. H. Svoboda, W. Denk, W. Knox, and S. Tsuda, "Twophoton laser scanning fluorescence microscopy using an diode-pumped Cr:LiSAF laser mode-locked with a saturable Bragg reflector," Opt. Lett. 1996 (in press).

129. P. F. Curley, A. I. Ferguson, J. G. White, and W. B. Amos, "Application of a femtosecond self-sustaining modelocked Ti:Sapphire laser to the field of laser scanning confocal microscopy," Opt. Quant. Electr. 24, 851-859 (1992). 